\title{
COMPLETE MONOTONICITY OF A FUNCTION INVOLVING THE GAMMA FUNCTION AND APPLICATIONS
}

\author{
FENG QI
}

\begin{abstract}
In the article we present necessary and sufficient conditions for a function involving the logarithm of the gamma function to be completely monotonic and apply these results to bound the gamma function $\Gamma(x)$, the $n$-th harmonic number $\sum_{k=1}^{n} \frac{1}{k}$, and the factorial $n$ !.
\end{abstract}

\section{INTRODUCTION}

We recall from [18, Chapter XIII], [29, Chapter 1] and [31, Chapter IV] that a function $f$ is said to be completely monotonic on an interval $I$ if $f$ has derivatives of all orders on $I$ and

$$
0 \leq(-1)^{n} f^{(n)}(x)<\infty
$$

for $x \in I$ and $n \geq 0$.

We also recall from $[1$, p. 254, 6.1.1] that the classical Euler gamma function $\Gamma(x)$ may be defined by

$$
\Gamma(x)=\int_{0}^{\infty} t^{x-1} e^{-t} \mathrm{~d} t, \quad x>0 .
$$

The logarithmic derivative of $\Gamma(x)$, denoted by $\psi(x)=\frac{\Gamma^{\prime}(x)}{\Gamma(x)}$, is called the psi or di-gamma function, and the derivatives $\psi^{(i)}(x)$ for $i \in \mathbb{N}$ are respectively called the polygamma functions. They are a series of important special functions and have much extensive applications in many branches such as statistics, probability, number theory, theory of 0-1 matrices, graph theory, combinatorics, physics, engineering, and other mathematical sciences.

Using Hermite-Hadamard's inequality (see [26, 27, 28]), the double inequality

$$
\begin{aligned}
& \left(x-\frac{1}{2}\right)\left[\ln \left(x-\frac{1}{2}\right)-1\right]+\ln \sqrt{2 \pi}-\frac{1}{24(x-1)} \leq \ln \Gamma(x) \\
& \quad \leq\left(x-\frac{1}{2}\right)\left[\ln \left(x-\frac{1}{2}\right)-1\right]+\ln \sqrt{2 \pi}-\frac{1}{24\left(\sqrt{x^{2}+x+1 / 2}-1 / 2\right)}
\end{aligned}
$$

for $x>1$ was obtained in [4, p. 236, Theorem 1].

In [30, p. 1774, Theorem 2.3], the function

$$
H(x)=\ln \Gamma(x+1)-\left(x+\frac{1}{2}\right) \ln \left(x+\frac{1}{2}\right)+x+\frac{1}{2}-\frac{1}{2} \ln (2 \pi)+\frac{1}{24(x+1 / 2)}
$$

2010 Mathematics Subject Classification. Primary 26A48, 33B15; Secondary 26D15.

Key words and phrases. Complete monotonicity, logarithmically completely monotonic function, gamma function, inequality, necessary and sufficient condition, harmonic number, factorial, application.

The author was supported in part by the Science Foundation of Tianjin Polytechnic University. 
was proved to be completely monotonic on $(0, \infty)$. From this it was deduced in $[30$, p. 1775, Corollary 2.4] that the double inequality

$$
\begin{aligned}
\alpha\left(\frac{x+1 / 2}{e}\right)^{x+1 / 2} e^{-1 / 24(x+1 / 2)}<\Gamma(x+1) & \\
& \leq \beta\left(\frac{x+1 / 2}{e}\right)^{x+1 / 2} e^{-1 / 24(x+1 / 2)}
\end{aligned}
$$

holds for $x>0$, where $\alpha=\sqrt{2 \pi}=2.50 \cdots$ and $\beta=\sqrt{2} e^{7 / 12}=2.53 \cdots$ are the best possible constants.

We observe that, by taking the natural exponentials on all sides of (1.3) and replacing $x$ by $x+1$, the inequality (1.3) may be rewritten as

$$
\begin{aligned}
& \sqrt{2 \pi}\left(\frac{x+1 / 2}{e}\right)^{x+1 / 2} e^{-1 / 24 x}<\Gamma(x+1) \\
& \leq \sqrt{2 \pi}\left(\frac{x+1 / 2}{e}\right)^{x+1 / 2} e^{-1 / 24\left(\sqrt{x^{2}+3 x+5 / 2}-1 / 2\right)}, \quad x>0 .
\end{aligned}
$$

Hence, it is clear that the left hand side inequality in (1.5) is stronger than the corresponding one in (1.3) or, equivalently, (1.6). But, when $x \geq 1$, the right hand side inequality in (1.5) is weaker than the corresponding one in (1.3) or, equivalently, (1.6).

For $\lambda \geq 0$ and $x \in(0, \infty)$, let

$$
H_{\lambda}(x)=\ln \Gamma(x+1)-\left(x+\frac{1}{2}\right) \ln \left(x+\frac{1}{2}\right)+x+\frac{1}{2}-\ln \sqrt{2 \pi}+\frac{1}{24(x+\lambda)} .
$$

The first aim of this paper is to find necessary and sufficient conditions for the functions $\pm H_{\lambda}(x)$ to be completely monotonic on $(0, \infty)$. The second aim is to apply the complete monotonicity of $\pm H_{\lambda}(x)$ to establish inequalities for bounding the gamma function, the $n$-th harmonic number $\sum_{k=1}^{n} \frac{1}{k}$, and the factorial $n$ !.

\section{NECESSARY AND SUFFICIENT CONDITIONS}

Our main results are necessary and sufficient conditions for the functions $\pm H_{\lambda}(x)$ to be completely monotonic on $(0, \infty)$, which can be stated in the following theorem.

Theorem 1. For $x \in(0, \infty)$ and $\lambda \geq 0$,

(1) if and only if $0 \leq \lambda \leq \frac{1}{2}$, the function $H_{\lambda}(x)$ is completely monotonic;

(2) if

$$
\lambda \geq-\inf _{t \in(0, \infty)}\left\{\frac{1}{t} \ln \left[\frac{24}{t^{2}}\left(\frac{1}{e^{t / 2}}-\frac{t}{e^{t}-1}\right)\right]\right\},
$$

the function $-H_{\lambda}(x)$ is completely monotonic;

(3) if $\lambda \geq \frac{3}{2}$, a special case of the inequality (2.1), the function $-H_{\lambda}(x)$ is completely monotonic on $(0, \infty)$.

Proof of the necessary condition in (1) of Theorem 1. If the function $H_{\lambda}(x)$ is completely monotonic on $(0, \infty)$ for $\lambda \geq 0$, then $H_{\lambda}(x) \geq 0$, which may be rearranged as

$$
\lambda \leq-x-\frac{1}{24 f(x)}
$$


where

$$
f(x)=\ln \Gamma(x+1)-\left(x+\frac{1}{2}\right) \ln \left(x+\frac{1}{2}\right)+x+\frac{1}{2}-\frac{1}{2} \ln (2 \pi), \quad x>0 .
$$

Since

$$
\ln \Gamma(z) \sim\left(z-\frac{1}{2}\right) \ln z-z+\frac{1}{2} \ln (2 \pi)+\frac{1}{12 z}-\frac{1}{360 z^{3}}+\frac{1}{1260 z^{5}}-\cdots
$$

as $z \rightarrow \infty$ in $|\arg z|<\pi$, see [1, p. 257,6.1.41], we have

$$
f(x) \sim\left(x+\frac{1}{2}\right) \ln \frac{x+1}{x+1 / 2}-\frac{1}{2}+\frac{1}{12(x+1)}+O\left(\frac{1}{x^{2}}\right)
$$

as $x \rightarrow \infty$. As a result, we have

$$
-x-\frac{1}{24 f(x)} \sim-\frac{1}{2} \cdot \frac{h_{1}(x)}{h_{2}(x)}
$$

as $x \rightarrow \infty$, where

$$
h_{1}(x)=12 x\left(2 x^{2}+3 x+1\right) \ln \frac{x+1}{x+1 / 2}-12 x^{2}-9 x+1+O(1) \rightarrow \frac{1}{2}
$$

and

$$
h_{2}(x)=6\left(2 x^{2}+3 x+1\right) \ln \frac{x+1}{x+1 / 2}-6 x-5+O\left(\frac{1}{x}\right) \rightarrow-\frac{1}{2}
$$

as $x \rightarrow \infty$. So

$$
-x-\frac{1}{24 f(x)} \rightarrow \frac{1}{2}, \quad x \rightarrow \infty .
$$

This means that the necessary condition for the function $H_{\lambda}(x)$ to be completely monotonic on $(0, \infty)$ is $\lambda \leq \frac{1}{2}$.

First proof of the sufficient condition in (1) of Theorem 1. When $\lambda<\frac{1}{2}$, the function $H_{\lambda}(x)$ is

$$
\begin{aligned}
H_{\lambda}(x) & =H_{1 / 2}(x)+\frac{1}{24(x+\lambda)}-\frac{1}{24(x+1 / 2)} \\
& =H_{1 / 2}(x)+\frac{1}{24} \int_{\lambda}^{1 / 2} \frac{1}{(x+t)^{2}} \mathrm{~d} t .
\end{aligned}
$$

Since the function $H_{1 / 2}(x)=H(x)$ is completely monotonic on $(0, \infty)$, see [30, p. 1774, Theorem 2.3], and the integral $\int_{\lambda}^{1 / 2} \frac{1}{(x+t)^{2}} \mathrm{~d} t$ is clearly completely monotonic on $(-\lambda, \infty) \supset(0, \infty)$, also since the product and sum of finite completely monotonic functions are completely monotonic on their common domain, it follows immediately that the function $H_{\lambda}(x)$ is completely monotonic on $(0, \infty)$ if $\lambda \leq \frac{1}{2}$.

Second proof of the sufficient condition in (1) of Theorem 1. The famous Binet's first formula of $\ln \Gamma(x)$ for $x>0$ is given by

$$
\ln \Gamma(x)=\left(x-\frac{1}{2}\right) \ln x-x+\ln \sqrt{2 \pi}+\theta(x),
$$

where

$$
\theta(x)=\int_{0}^{\infty}\left(\frac{1}{e^{t}-1}-\frac{1}{t}+\frac{1}{2}\right) \frac{e^{-x t}}{t} \mathrm{~d} t
$$


for $x>0$ is called the remainder of Binet's first formula for the logarithm of the gamma function, see $[17$, p. 11] or $[25$, p. 462]. The formulas

$$
\Gamma(z)=k^{z} \int_{0}^{\infty} t^{z-1} e^{-k t} \mathrm{~d} t
$$

and

$$
\ln \frac{b}{a}=\int_{0}^{\infty} \frac{e^{-a u}-e^{-b u}}{u} \mathrm{~d} u
$$

for $\operatorname{Re} z>0, \operatorname{Re} k>0, a>0$ and $b>0$ can be found in [1, p. 255, 6.1.1 and p. 230, 5.1.32]. Utilizing these formulas yields

$$
\begin{aligned}
f(x) & =\left(x+\frac{1}{2}\right) \ln \frac{x}{x+1 / 2}+\frac{1}{2}+\theta(x), \\
f^{\prime}(x) & =\frac{1}{2 x}+\ln \frac{x}{x+1 / 2}+\theta^{\prime}(x) \\
& =\int_{0}^{\infty}\left(\frac{e^{-t / 2}}{t}-\frac{1}{e^{t}-1}\right) e^{-x t} \mathrm{~d} t, \\
H_{\lambda}^{\prime}(x) & =f^{\prime}(x)-\frac{1}{24(x+\lambda)^{2}} \\
& =\int_{0}^{\infty}\left(\frac{e^{-t / 2}}{t}-\frac{1}{e^{t}-1}-\frac{t e^{-\lambda t}}{24}\right) e^{-x t} \mathrm{~d} t .
\end{aligned}
$$

From (1.7), (2.2) and (2.9), it is easy to see that

$$
\lim _{x \rightarrow \infty} H_{\lambda}(x)=\lim _{x \rightarrow \infty} f(x)+\lim _{x \rightarrow \infty} \frac{1}{24(x+\lambda)}=0 .
$$

Therefore, in order to prove the complete monotonicity of $\mp H_{\lambda}(x)$, it suffices to show $\pm H_{\lambda}^{\prime}(x)$ is completely monotonic on $(0, \infty)$. For this, it is sufficient to have

$$
\frac{e^{-t / 2}}{t}-\frac{1}{e^{t}-1}-\frac{t e^{-\lambda t}}{24} \gtreqless 0
$$

for all $t \in(0, \infty)$, which is equivalent to

$$
\lambda \gtreqless-\frac{1}{t} \ln \left[\frac{24}{t}\left(\frac{e^{-t / 2}}{t}-\frac{1}{e^{t}-1}\right)\right]
$$

for all $t \in(0, \infty)$.

We claim that

$$
-\frac{1}{t} \ln \left[\frac{24}{t}\left(\frac{e^{-t / 2}}{t}-\frac{1}{e^{t}-1}\right)\right] \geq \frac{1}{2}
$$

for $t \in(0, \infty)$. In fact, this inequality can be reduced to

$$
\frac{24}{t}\left(\frac{e^{-t / 2}}{t}-\frac{1}{e^{t}-1}\right) \leq e^{-t / 2}
$$

equivalently,

$$
\left(t^{2}-24\right) e^{t}+24 t e^{t / 2}-t^{2}+24=\sum_{k=5}^{\infty}\left\{[k(k-1)-24] 2^{k}+48 k\right\} \frac{t^{k}}{k ! 2^{k}}
$$




$$
\begin{aligned}
& =\frac{7}{24} t^{5}+\sum_{k=6}^{\infty}\left\{[k(k-1)-24] 2^{k}+48 k\right\} \frac{t^{k}}{k ! 2^{k}} \\
& \geq \frac{7}{24} t^{5}+\sum_{k=6}^{\infty}\{[k(k-1)-24](1+k)+48 k\} \frac{t^{k}}{k ! 2^{k}} \\
& \geq \frac{7}{24} t^{5}+\sum_{k=6}^{\infty}\left(k^{3}+23 k-24\right) \frac{t^{k}}{k ! 2^{k}} \\
& \geq 0 .
\end{aligned}
$$

Thus, when $0 \leq \lambda \leq \frac{1}{2}$, the function $H_{\lambda}(x)$ is completely monotonic on $(0, \infty)$.

Proof of (2) in Theorem 1. This follows from the inequality with the sign $\geq$ in (2.13).

Proof of (3) in Theorem 1. Suppose that

$$
-\frac{1}{t} \ln \left[\frac{24}{t}\left(\frac{e^{-t / 2}}{t}-\frac{1}{e^{t}-1}\right)\right] \leq \lambda
$$

for $t \in(0, \infty)$. Then

$$
\frac{24}{t}\left(\frac{e^{-t / 2}}{t}-\frac{1}{e^{t}-1}\right) \geq e^{-\lambda t}
$$

which can be rewritten as

$$
24 e^{\lambda t}\left(e^{t}-t e^{t / 2}-1\right) \geq t^{2} e^{t / 2}\left(e^{t}-1\right) .
$$

Expanding at $t=0$ the functions on both sides of the above inequality into power series yields

$$
24 \sum_{k=3}^{\infty}\left[(\lambda+1)^{k}-\lambda^{k}-k\left(\lambda+\frac{1}{2}\right)^{k-1}\right] \frac{t^{k}}{k !} \geq \sum_{k=3}^{\infty}\left[\left(\frac{3}{2}\right)^{k-2}-\left(\frac{1}{2}\right)^{k-2}\right] \frac{t^{k}}{(k-2) !} .
$$

Let $h_{k ; \lambda}(u)=(\lambda+u)^{k-1}$. Then, by the left hand side inequality in the double integral inequality

$$
\frac{(b-a)^{2}}{24} m \leq \frac{1}{b-a} \int_{a}^{b} f(t) \mathrm{d} t-f\left(\frac{a+b}{2}\right) \leq \frac{(b-a)^{2}}{24} M,
$$

where $f:[a, b] \rightarrow \mathbb{R}$ be a twice differentiable mapping and $m \leq f^{\prime \prime}(t) \leq M$ for all $t \in(a, b)$, see $[5,6]$ and $[26$, p. 236, Theorem A], we obtain

$$
\begin{aligned}
(\lambda+1)^{k}-\lambda^{k}-k\left(\lambda+\frac{1}{2}\right)^{k-1} & =k\left[\frac{1}{1-0} \int_{0}^{1} h_{k ; \lambda}(u) \mathrm{d} u-h_{k ; \lambda}\left(\frac{0+1}{2}\right)\right] \\
& \geq k \cdot \frac{(1-0)^{2}}{24} \inf _{u \in(0,1)} h_{k ; \lambda}^{\prime \prime}(u) \\
& =\frac{1}{24} k(k-1)(k-2) \lambda^{k-3} .
\end{aligned}
$$

Therefore, in order to have the inequality (2.15) hold, it is sufficient to make

$$
(k-2) \lambda^{k-3} \geq\left(\frac{3}{2}\right)^{k-2}-\left(\frac{1}{2}\right)^{k-2},
$$


which is equivalent to

$$
\lambda \geq \sqrt[k-3]{\frac{1}{k-2}\left[\left(\frac{3}{2}\right)^{k-2}-\left(\frac{1}{2}\right)^{k-2}\right]}
$$

for all $k \geq 3$. Since

$$
\sqrt[k-3]{\frac{1}{k-2}\left[\left(\frac{3}{2}\right)^{k-2}-\left(\frac{1}{2}\right)^{k-2}\right]} \leq \sqrt[k-3]{\frac{1}{k-2}\left(\frac{3}{2}\right)^{k-2}} \rightarrow \frac{3}{2}
$$

as $k \rightarrow \infty$, it follows that when $\lambda \geq \frac{3}{2}$ the inequality (2.15) holds. This means that when $\lambda \geq \frac{3}{2}$ the negative of the function (2.11) is completely monotonic on $(0, \infty)$. The proof of (2) in Theorem 1 is complete.

\section{Applications}

In this section, we apply the complete monotonicity of $\pm H_{\lambda}(x)$ to establish inequalities for bounding the gamma function, the $n$-th harmonic number $\sum_{k=1}^{n} \frac{1}{k}$, and the factorial $n$ !.

Theorem 2. For $x \in(0, \infty)$, the gamma function $\Gamma(x+1)$ can be bounded by

$$
\begin{aligned}
& \sqrt{2 \pi}\left(\frac{x+1 / 2}{e}\right)^{x+1 / 2} \exp \left(-\frac{1}{24(x+1 / 2)}\right)<\Gamma(x+1) \\
&<\sqrt{2 \pi}\left(\frac{x+1 / 2}{e}\right)^{x+1 / 2} \exp \left(\frac{1}{24}\left(\frac{2 x}{x+1 / 2}-12(\ln \pi-1)\right)\right)
\end{aligned}
$$

and

$$
\begin{aligned}
\sqrt{2 \pi}\left(\frac{x+1 / 2}{e}\right)^{x+1 / 2} \exp ( & \left.\frac{1}{24}\left[\frac{2 x}{3(x+3 / 2)}-12(\ln \pi-1)\right]\right)<\Gamma(x+1) \\
& <\sqrt{2 \pi}\left(\frac{x+1 / 2}{e}\right)^{x+1 / 2} \exp \left(-\frac{1}{24(x+3 / 2)}\right) .
\end{aligned}
$$

Proof. By (2.4), it is easy to see that

$$
\lim _{x \rightarrow \infty} H_{\lambda}(x)=0 .
$$

Moreover, it is immediate that

$$
\lim _{x \rightarrow 0^{+}} H_{\lambda}(x)=\frac{1}{24}\left(\frac{1}{\lambda}+12-12 \ln \pi\right) .
$$

By Theorem 1, it readily follows that when and only when $0 \leq \lambda \leq \frac{1}{2}$ the function $H_{\lambda}(x)$ is decreasing on $(0, \infty)$. So, we have

$$
0<H_{\lambda}(x)<\frac{1}{24}\left(\frac{1}{\lambda}+12-12 \ln \pi\right)
$$

that is,

$$
\begin{aligned}
& \sqrt{2 \pi}\left(\frac{x+1 / 2}{e}\right)^{x+1 / 2} \exp \left(-\frac{1}{24(x+\lambda)}\right)<\Gamma(x+1) \\
& <\sqrt{2 \pi}\left(\frac{x+1 / 2}{e}\right)^{x+1 / 2} \exp \left(\frac{1}{24}\left(\frac{1}{\lambda}+12-12 \ln \pi-\frac{1}{x+\lambda}\right)\right)
\end{aligned}
$$


for $0 \leq \lambda \leq \frac{1}{2}$ and $x \in(0, \infty)$. The inequality (3.1) is proved.

Similarly, when $\lambda \geq \frac{3}{2}$, the function $H_{\lambda}(x)$ is increasing on $(0, \infty)$, and the inequality (3.4) is reversed on $(0, \infty)$ for $\lambda \geq \frac{3}{2}$. The inequality (3.2) follows.

Theorem 3. For $n \in \mathbb{N}$, the $n$-th harmonic number $\sum_{k=1}^{n} \frac{1}{k}$ can be bounded by

$$
\begin{aligned}
\ln \left(n+\frac{1}{2}\right)+\frac{1}{24(n+1 / 2)^{2}}+1-\ln \frac{3}{2} & -\frac{1}{54} \leq \sum_{k=1}^{n} \frac{1}{k} \\
& <\ln \left(n+\frac{1}{2}\right)+\frac{1}{24(n+1 / 2)^{2}}+\gamma
\end{aligned}
$$

and

$$
\begin{aligned}
\ln \left(n+\frac{1}{2}\right)+\frac{1}{24(n+3 / 2)^{2}} & +\gamma<\sum_{k=1}^{n} \frac{1}{k} \\
\leq & \ln \left(n+\frac{1}{2}\right)+\frac{1}{24(n+3 / 2)^{2}}+1-\ln \frac{3}{2}-\frac{1}{90},
\end{aligned}
$$

where $\gamma=0.577 \cdots$ stands for Euler-Mascheroni's constant.

Proof. By Theorem 1 and the definition of completely monotonic functions, it follows that

(1) when $0 \leq \lambda \leq \frac{1}{2}$, the function $H_{\lambda}^{\prime}(x)$ is increasing on $(0, \infty)$,

(2) when $\lambda \geq \frac{3}{2}$, the function $H_{\lambda}^{\prime}(x)$ is decreasing on $(0, \infty)$. Since

$$
H_{\lambda}^{\prime}(x)=\psi(x+1)-\ln \left(x+\frac{1}{2}\right)-\frac{1}{24(x+\lambda)^{2}}
$$

and $\lim _{x \rightarrow \infty} H_{\lambda}^{\prime}(x)=0$, it follows readily that

$$
\begin{aligned}
\ln \left(x+\frac{1}{2}\right)+\frac{1}{24(x+1 / 2)^{2}}+1-\gamma-\ln \frac{3}{2} & -\frac{1}{54} \leq \psi(x+1) \\
& <\ln \left(x+\frac{1}{2}\right)+\frac{1}{24(x+1 / 2)^{2}}
\end{aligned}
$$

and

$$
\begin{aligned}
\ln \left(x+\frac{1}{2}\right)+\frac{1}{24(x+3 / 2)^{2}} & <\psi(x+1) \\
\leq & \ln \left(x+\frac{1}{2}\right)+\frac{1}{24(x+3 / 2)^{2}}+1-\gamma-\ln \frac{3}{2}-\frac{1}{90} .
\end{aligned}
$$

for $x \in[1, \infty)$. Taking $x=n$ and using

$$
\psi(n+1)=\sum_{k=1}^{n} \frac{1}{k}-\gamma,
$$

see [1, p. 258, 6.3.2], in (3.7) and (3.8) give inequalities (3.5) and (3.6).

We recall from $[2,22]$ that a function $f$ is said to be logarithmically completely monotonic on an interval $I \subseteq \mathbb{R}$ if it has derivatives of all orders on $I$ and its logarithm $\ln f$ satisfies

$$
(-1)^{k}[\ln f(x)]^{(k)} \geq 0
$$


for $k \in \mathbb{N}$ on $I$.

Theorem 4. For $x \in(0, \infty)$ and $\lambda \geq 0$, let

$$
G_{\lambda}(x)=\frac{e^{x} \Gamma(x+1)}{(x+1 / 2)^{x+1 / 2}} \exp \frac{1}{24(x+\lambda)} .
$$

Then the function $G_{\lambda}(x)$ has the following properties:

(1) if and only if $0 \leq \lambda \leq \frac{1}{2}$, the function $G_{\lambda}(x)$ is logarithmically completely monotonic on $(0, \infty)$;

(2) if the inequality (2.1) is valid, the reciprocal of the function $G_{\lambda}(x)$ is logarithmically completely monotonic on $(0, \infty)$;

(3) if $\lambda \geq \frac{3}{2}$, a special case of the inequality (2.1), the reciprocal of the function $G_{\lambda}(x)$ is logarithmically completely monotonic on $(0, \infty)$.

Proof. This follows from the obvious fact that

$$
\ln G_{\lambda}(x)=H_{\lambda}(x)-\frac{1-\ln (2 \pi)}{2}
$$

and the definition of logarithmically completely monotonic functions.

Theorem 5. For $n \in \mathbb{N}$, the factorial $n$ ! can be bounded by

$$
\begin{aligned}
& \sqrt{2 \pi}\left(\frac{n+1 / 2}{e}\right)^{n+1 / 2} \exp \left(-\frac{1}{24(n+1 / 2)}\right)<n ! \\
& \leq \sqrt{2 \pi}\left(\frac{n+1 / 2}{e}\right)^{n+1 / 2} \exp \left(\frac{1}{24}\left[12\left(3-\ln \pi+\ln \frac{4}{27}\right)-\frac{1}{3(n+1 / 2)}\right]\right)
\end{aligned}
$$

and

$$
\begin{aligned}
\sqrt{2 \pi}\left(\frac{n+1 / 2}{e}\right)^{n+1 / 2} \exp & \left(\frac{1}{24}\left[12\left(3-\ln \pi+\ln \frac{4}{27}\right)+\frac{1}{5(n+3 / 2)}\right]\right) \leq n ! \\
& <\sqrt{2 \pi}\left(\frac{n+1 / 2}{e}\right)^{n+1 / 2} \exp \left(-\frac{1}{24(n+3 / 2)}\right) .
\end{aligned}
$$

Proof. Combining (3.3) and

$$
H_{\lambda}(1)=\frac{1}{24}\left[\frac{1}{\lambda+1}+36-12 \ln (2 \pi)-36 \ln \frac{3}{2}\right],
$$

with Theorem 1 and the proof of Theorem 2 reveals

$$
\begin{aligned}
& \sqrt{2 \pi}\left(\frac{x+1 / 2}{e}\right)^{x+1 / 2} \exp \left(-\frac{1}{24(x+1 / 2)}\right)<\Gamma(x+1) \\
& \leq \sqrt{2 \pi}\left(\frac{x+1 / 2}{e}\right)^{x+1 / 2} \exp \left(\frac{1}{24}\left[12\left(3-\ln \pi+\ln \frac{4}{27}\right)-\frac{1}{3(x+1 / 2)}\right]\right)
\end{aligned}
$$

and

$$
\begin{array}{r}
\sqrt{2 \pi}\left(\frac{x+1 / 2}{e}\right)^{x+1 / 2} \exp \left(\frac{1}{24}\left[12\left(3-\ln \pi+\ln \frac{4}{27}\right)+\frac{1}{5(x+3 / 2)}\right]\right) \leq \\
\Gamma(x+1)<\sqrt{2 \pi}\left(\frac{x+1 / 2}{e}\right)^{x+1 / 2} \exp \left(-\frac{1}{24(x+3 / 2)}\right) .
\end{array}
$$


Letting $x=n$ and using $\Gamma(n+1)=n$ ! in (3.14) and (3.15) leads to inequalities (3.12) and (3.13). The proof is complete.

\section{REMARKS}

In this section, we would like to comment some results above-presented.

Remark 1. The inequality (2.14) and the inequality (2.15) for $\lambda \geq \frac{3}{2}$ may be rearranged as the double inequality

$$
\frac{1}{e^{x / 2}}-\frac{x^{2}}{24 e^{\beta x}} \leq \frac{x}{e^{x}-1} \leq \frac{1}{e^{x / 2}}-\frac{x^{2}}{24 e^{\alpha x}}
$$

on $(0, \infty)$, where $\alpha=\frac{3}{2}$ and $\beta=\frac{1}{2}$. This improves the right hand side and partially improves the left hand side of the double inequality

$$
\frac{1}{e^{x}}<\frac{x}{e^{x}-1}<\frac{1}{e^{x / 2}}, \quad x>0
$$

in [23, p. 2550, Proposition 4.1].

We guess that the scalar $\alpha=\frac{3}{2}$ in (4.1) can be replaced by a smaller number, for example, 1 , but the constant $\beta=\frac{1}{2}$ in (4.1) is the best possible.

In [19], some related inequalities for the exponential function $e^{x}$ were constructed.

In a subsequent paper, we will refine the right hand side of the double inequality (4.1) and employ it to strengthen double inequalities for bounding Mathieu's series

$$
S(r)=\sum_{n=1}^{\infty} \frac{2 n}{\left(n^{2}+r^{2}\right)^{2}}, \quad r>0
$$

and the like. For more information on bounding Mathieu type series, please refer to $[16,20,23]$ and closely related references therein.

Remark 2. There have been plenty of references devoted to bounding the $n$-th harmonic number $\sum_{k=1}^{n} \frac{1}{k}$ for $n \in \mathbb{N}$, for example, [7, 10,11,24] and closely related references therein.

Remark 3. Several inequalities for bounding the gamma function were also established and collected in $[9,12,13]$. See also [21, pp. 52-57] and lots of references cited therein.

Remark 4. It was proved once again in $[3,8,22]$ that the set of logarithmically completely monotonic functions is a subset of the completely monotonic functions. This implies that Theorem 4 is not trivial.

Remark 5. In [14, 15], it was shown that the function

$$
g_{\beta}(x)=\frac{e^{x} \Gamma(x+1)}{(x+\beta)^{x+\beta}}
$$

on the interval $(\max \{0,-\beta\}, \infty)$ for $\beta \in \mathbb{R}$ is logarithmically completely monotonic if and only if $\beta \geq 1$ and that the function $\left[g_{\alpha, \beta}(x)\right]^{-1}$ is logarithmically completely monotonic if and only if $\beta \leq \frac{1}{2}$. See also [21, pp. 53-54, Section 5.6]. Motivated by this and Theorem 4, we would like to ask a question: How about the logarithmically complete monotonicity of the function

$$
G_{\lambda, \mu}(x)=\frac{e^{x} \Gamma(x+1)}{(x+\mu)^{x+\mu}} \exp \frac{1}{24(x+\lambda)}
$$

on the interval $(\max \{0,-\lambda,-\mu\}, \infty)$ ? where $\lambda$ and $\mu$ are given real numbers. 


\section{REFERENCES}

[1] M. Abramowitz and I. A. Stegun (Eds), Handbook of Mathematical Functions with Formulas, Graphs, and Mathematical Tables, National Bureau of Standards, Applied Mathematics Series 55, 4th printing, with corrections, Washington, 1965. 1, 3, 4, 7

[2] R. D. Atanassov and U. V. Tsoukrovski, Some properties of a class of logarithmically completely monotonic functions, C. R. Acad. Bulgare Sci. 41 (1988), no. 2, 21-23. 7

[3] C. Berg, Integral representation of some functions related to the gamma function, Mediterr. J. Math. 1 (2004), no. 4, 433-439. 9

[4] J. Bukac, T. Burić and N. Elezović, Stirling's formula revisited via some classical and new inequalities, Math. Inequal. Appl. 14 (2011), no. 1, 235-245. 1

[5] P. Cerone and S. S. Dragomir, Midpoint-type rules from an inequality point of view, Handbook of Analytic-Computational Methods in Applied Mathematics, Editor: G. Anastassiou, CRC Press, New York, 2000, 135-200. 5

[6] P. Cerone and S. S. Dragomir, Trapezoidal-type rules from an inequality point of view, Handbook of Analytic-Computational Methods in Applied Mathematics, Editor: G. Anastassiou, CRC Press, New York, 2000, 65-134. 5

[7] Ch.-P. Chen and F. Qi, The best bounds of the $n$-th harmonic number, Glob. J. Appl. Math. Math. Sci. 1 (2008), no. 1, 41-49. 9

[8] B.-N. Guo and F. Qi, A property of logarithmically absolutely monotonic functions and the logarithmically complete monotonicity of a power-exponential function, Politehn. Univ. Bucharest Sci. Bull. Ser. A Appl. Math. Phys. 72 (2010), no. 2, 21-30. 9

[9] B.-N. Guo and F. Qi, A refinement of a double inequality for the gamma function, Publ. Math. Debrecen 79 (2011), in press. 9

[10] B.-N. Guo and F. Qi, Sharp bounds for harmonic numbers, Appl. Math. Comput. ?? (2011), no. ??, in press; Available online at http://dx.doi.org/10.1016/j.amc.2011.01.089. 9

[11] B.-N. Guo and F. Qi, Some properties of the psi and polygamma functions, Hacet. J. Math. Stat. 39 (2010), no. 2, 219-231. 9

[12] B.-N. Guo and F. Qi, Two new proofs of the complete monotonicity of a function involving the psi function, Bull. Korean Math. Soc. 47 (2010), no. 1, 103-111; Available online at http://dx.doi.org/10.4134/BKMS.2010.47.1.103. 9

[13] B.-N. Guo, Y.-J. Zhang and F. Qi, Refinements and sharpenings of some double inequalities for bounding the gamma function, J. Inequal. Pure Appl. Math. 9 (2008), no. 1, Art. 17; Available online at http://www.emis.de/journals/JIPAM/article953.html?sid=953. 9

[14] S. Guo, Some classes of completely monotonic functions involving the gamma function, Internat. J. Pure Appl. Math. 30 (2006), no. 4, 561-566. 9

[15] S. Guo, F. Qi and H. M. Srivastava, Necessary and sufficient conditions for two classes of functions to be logarithmically completely monotonic, Integral Transforms Spec. Funct. 18 (2007), no. 11, 819-826; Available online at http://dx.doi.org/10.1080/ 10652460701528933. 9

[16] A. Hoorfar and F. Qi, Some new bounds for Mathieu's series, Abstr. Appl. Anal. 2007 (2007), Article ID 94854, 10 pages; Available online at http://dx.doi.org/10.1155/2007/94854. 9

[17] W. Magnus, F. Oberhettinger and R. P. Soni, Formulas and Theorems for the Special Functions of Mathematical Physics, Springer, Berlin, 1966. 4

[18] D. S. Mitrinović, J. E. Pečarić and A. M. Fink, Classical and New Inequalities in Analysis, Kluwer Academic Publishers, Dordrecht/Boston/London, 1993. 1

[19] F. Qi, A method of constructing inequalities about $e^{x}$, Univ. Beograd. Publ. Elektrotehn. Fak. Ser. Mat. 8 (1997), 16-23. 9

[20] F. Qi, An integral expression and some inequalities of Mathieu type series, Rostock. Math. Kolloq. 58 (2004), 37-46. 9

[21] F. Qi, Bounds for the ratio of two gamma functions, J. Inequal. Appl. 2010 (2010), Article ID 493058, 84 pages; Available online at http://dx.doi.org/10.1155/2010/493058. 9

[22] F. Qi and Ch.-P. Chen, A complete monotonicity property of the gamma function, J. Math. Anal. Appl. 296 (2004), no. 2, 603-607. 7, 9

[23] F. Qi, Ch.-P. Chen and B.-N. Guo, Notes on double inequalities of Mathieu's series, Int. J. Math. Math. Sci. 2005 (16) (2005), 2547-2554; Available online at http://dx.doi.org/10. 1155/IJMMS . 2005.2547. 9 
[24] F. Qi, R.-Q. Cui, Ch.-P. Chen and B.-N. Guo, Some completely monotonic functions involving polygamma functions and an application, J. Math. Anal. Appl. 310 (2005), no. 1, 303-308. 9

[25] F. Qi and B.-N. Guo, Some properties of extended remainder of Binet's first formula for logarithm of gamma function, Math. Slovaca 60 (2010), no. 4, 461-470; Available online at http://dx.doi.org/10.2478/s12175-010-0025-7. 4

[26] F. Qi, Z.-L. Wei and Q. Yang, Generalizations and refinements of Hermite-Hadamard's inequality, Rocky Mountain J. Math. 35 (2005), no. 1, 235-251. 1, 5

[27] F. Qi and M.-L. Yang, Comparisons of two integral inequalities with Hermite-HadamardJensen's integral inequality, Internat. J. Appl. Math. Sci. 3 (2006), no. 1, 83-88. 1

[28] F. Qi and M.-L. Yang, Comparisons of two integral inequalities with Hermite-HadamardJensen's integral inequality, Octogon Math. Mag. 14 (2006), no. 1, 53-58. 1

[29] R. L. Schilling, R. Song and Z. Vondraček, Bernstein Functions, de Gruyter Studies in Mathematics 37, De Gruyter, Berlin, Germany, 2010. 1

[30] H. Şevli and N. Batır, Complete monotonicity results for some functions involving the gamma and polygamma functions, Math. Comput. Modelling 53 (2011), 1771-1775; Available online at http://dx.doi.org/10.1016/j.mcm.2010.12.055. 1, 2, 3

[31] D. V. Widder, The Laplace Transform, Princeton University Press, Princeton, 1946. 1

School of Mathematics and Informatics, Henan Polytechnic University, Jiaozuo City, Henan Province, 454010, China; Department of Mathematics, College of Science, Tianjin Polytechnic University, Tianjin City, 300160, China

E-mail address: qifeng618@gmail.com, qifeng618@hotmail.com, qifeng618@qq.com

$U R L:$ http://qifeng618. wordpress.com 